\title{
Failed Internal Jugular Vein Cannulation - Is Contrast-Enhanced Computed Tomography Mandatory?
}

\author{
Complications by central venous catheterization \\ Wolfram Schummer, MD, PhD, DEAA, EDIC ${ }^{1 *}$, Samir G Sakka, MD, PhD, DEAA, EDIC ${ }^{2}$ \\ ${ }^{I}$ HELIOS Spital Ueberlingen, Germany \\ ${ }^{2}$ Cologne Merheim, University Witten/Herdecke, Germany
}

*Corresponding Author: Wolfram Schummer, HELIOS Spital Ueberlingen, Germany.

Email: cwsm.schummer@gmx.de

\begin{abstract}
Still, arterial puncture during central venous catheterization occurs and may cause severe and potentially life-threatening complications. Here, we present three patients in whom severe complications requiring surgical or interventional procedures were required to stop cervical bleeding or occlude aneurysms spuria and re-establish compromised cerebral perfusion. Complications occurred even while ultrasound was used during cannulation attempts. With regard to the dimension and severity of sequelae which may develop after complicated puncture we would like to plead for a low threshold for performing contrast enhanced computed tomography.
\end{abstract}

Keywords: Central venous catheterization, arterial lesions, aneurysm, complications, computed tomography

\section{INTRODUCTION}

Still, despite increasing use of ultrasound complications by central venous catheterization in critically ill patients are common [1]. In particular, arterial vessel lacerations occur and may cause potentially lethal complications, especially in attempted internal jugular vein cannulation. Here, we present three patients with iatrogenic arterial injuries causing bleeding and/ or aneurysmatic lesions requiring interventional or surgical intervention.

\section{CASE 1}

A 79-year old woman required mechanical ventilation and vasopressor support after a general seizure on the normal ward. Computed tomography (CT) confirmed new cerebral infarction in the posterior area of the middle cerebral artery (MCA) territory. On the ICU, she underwent central venous catheterization while being dehydrated as visible by the low venous filling status. Despite using online-ultrasound, right internal jugular vein (IJV) could not be catheterized successfully. After vessel puncture, the guide wire could not be advanced and the procedure was stopped at this site. Finally, the right femoral vein was identified as adequate access site.

After removal of the endotracheal tube a few hours later, the patient developed swelling of the right neck and new neurological signs (i.e., rightsided arm paresis). She underwent a CT scan of the neck and thorax after i.v. injection of contrast medium. An active extravasation from a sidebranch of the Truncus thyrocervicalis was found and interpreted as an aneurysm spurium after unsuccessful catheterization (Fig. 1). A hematoma reaching the mediastinum causing compression of the esophagus was described. Selective angiography by trans-brachial access revealed a normo-caliber internal jugular vein and confirmed an approximately $5 \mathrm{~mm}$ large aneurysm (Fig. 2). The vessel damage was localized approximately $1.5 \mathrm{~cm}$ distal to the branching into the A. transversalis colli and was successfully occluded with 5 coils. Absence of any further extravasation could be confirmed by repeated imaging. Fortunately, neurological symptoms disappeared and the patient could be discharged from the ICU to the normal ward a few days later. 


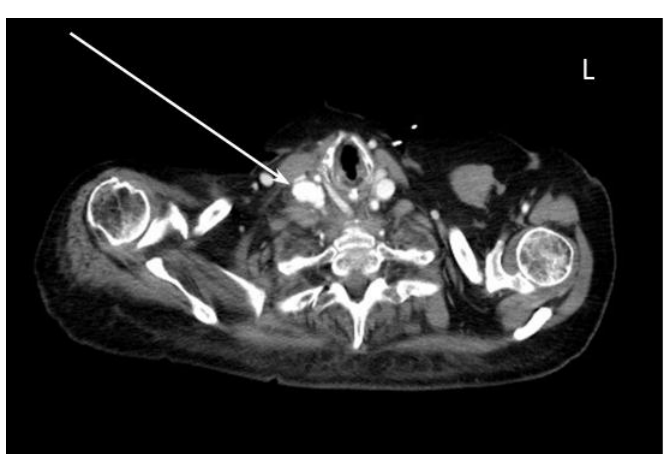

Figure 1. Computed tomography of the neck after i.v. $X$-ray contrast medium injection. The aneurysm of the A. thyrocervicalis and extravasation of the contrast medium are indicated by the arrow.

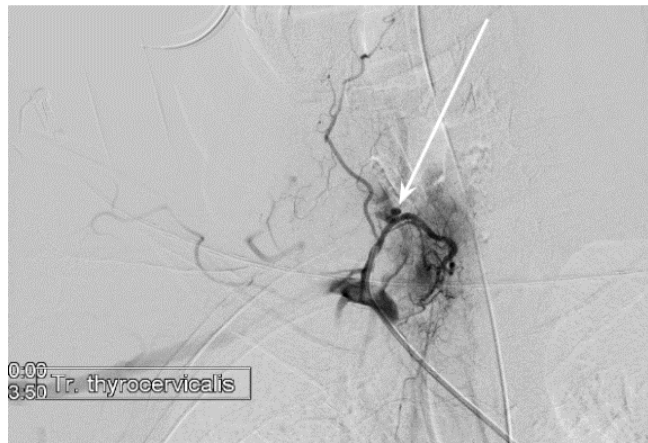

Figure 2. Selective angiography of the neck showing the aneurysm spurium (arrow)

\section{CASE 2}

A 78-year old woman who suffered from traumatic dens fracture underwent instrumented cervical spinal fusion in an external hospital. After surgery, the patient was awake and sufficiently breathing spontaneously, thus the endotracheal tube was removed in the operating theater (OR). After transfer to the ICU, a leftsided hemiparesis became obvious. A computed tomography (CT) scan revealed a cerebral infarction in the right middle cerebral artery (MCA) territory. First, it was argued that embolism might have occurred during cervical surgery. However, due to respiratory insufficiency the patient again was put on a respirator. Here, during laryngoscopy, the oropharynx was described as severely swollen and the endotracheal tube could only be placed with the help of difficult airway algorithm. In order to enable easier and safer weaning from mechanical ventilation, it was decided to perform a percutaneous tracheostomy. Ten days after neck surgery, the patient was successfully weaned from the respirator. However, at this stage a fulminant oropharyngeal bleeding occurred needing packing of the oropharynx. Then the patient was transferred to our hospital for consultation by an ENT physician. Examination of the oropharynx revealed a small mucosal lesion without any swelling. During the following 24 hours, however, the patient developed hemorrhagic shock, although no external bleeding was apparent.

A CT scan of head, neck and chest after i.v. injection of contrast medium showed a cervical hematoma with active bleeding from the internal carotid artery (Fig. 3). The lesion was covered by insertion of a stent. No further bleeding occurred. Two days later the patient could be transferred back to the referring hospital.

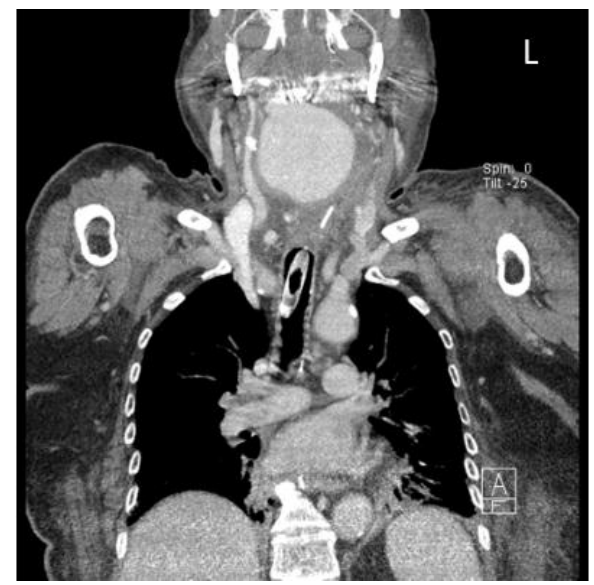

Figure 3. Computed tomography of the neck after i.v. $X$-ray contrast medium injection with an active bleeding

On request we were told that at the very early stages in this case right internal vein (IJV) cannulation had been attempted but failed. While unsuccessful at this site, the central venous catheter was successfully inserted contralaterally. However, no further details on the insertion procedure were available. Considering the change of insertion site it seems plausible to presume a difficult procedure at the right IJV and probably also an arterial puncture with the consequences described.

\section{CASE 3}

A 52-year old male developed decompensation of ischemic cardiomyopathy while waiting for heart transplantation. The patient was on dual antiplatelet therapy with acetylsalicylate and prasugrel and receiving a vitamin $\mathrm{K}$ antagonist. Since the patient required vasoactive support, he underwent central venous catheterization in the emergency department. The access procedure was done by an experienced operator, however, without ultrasound assistance. The first needle pass resulted in an arterial blood return. Due to a swiftly enlarging hematoma, the access procedure had to be stopped and local pressure was applied to the access site. However, increasing respiratory distress required urgent 
endotracheal intubation. Due to grotesque anatomic displacement of the larynx the first attempt failed (Fig. 4). An experienced anesthesiologist was able to narrowly secure the airway (with difficulties). The patient was transferred to the ICU where the team successfully treated shock and poor gas exchange. After stabilization, a contrastenhanced CT scan of the neck and thorax was performed showing two densities of the hematoma indicating an active arterial bleeding (Fig. 5). The patient underwent open surgery for removal of the hematoma. The inferior thyroid artery as one branch of the thyrocervical trunk which obviously had been punctured unintentionally was identified as source of bleeding. Unfortunately, the patient died three weeks later in multiple organ failure.

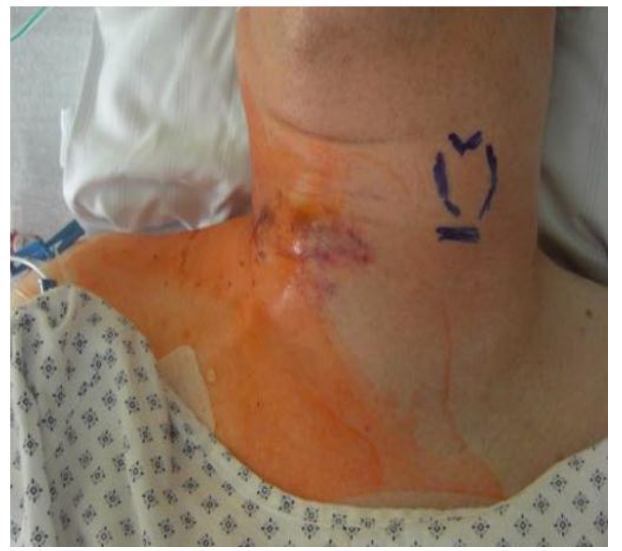

Figure 4. Photograph of the neck showing massive right-sided swelling and dislocation of anatomical structures to the left (larynx indicated on the skin)

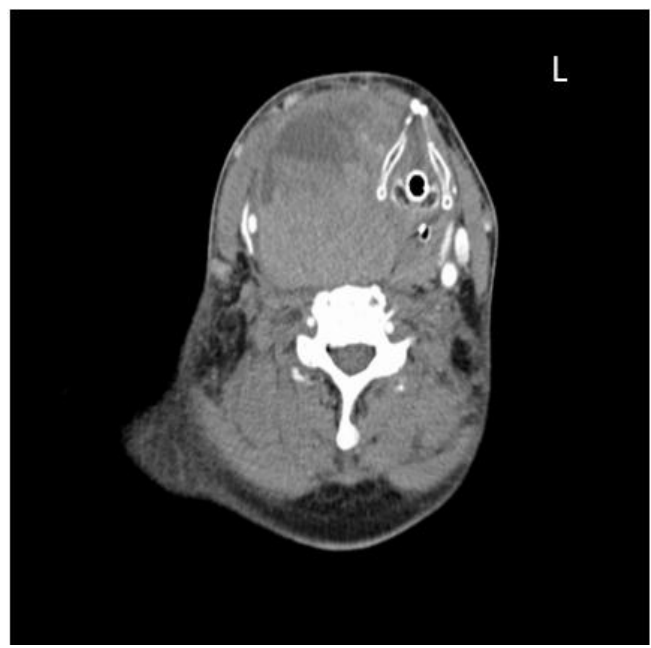

Figure 5. Computed tomography of the neck after i.v. $X$-ray contrast medium injection showing massive right-sided swelling and dislocation of the larynx

\section{DISCUSSION}

Our case series demonstrates that while seeking vascular access by the internal jugular vein unintentional arterial punctures still occur [1].
However, this may occur even under ultrasound guidance. However, with ultrasound guidance this risk of course seems to be substantially minimized [2].

Unintentional arterial puncture may result in formation of hematoma and/ or an aneurysm spurium. In case 1 it is sure and in case 2 it seems reasonable that an aneurysm spurium developed after arterial puncture. However, in case 2 the surgical procedure itself may have been responsible for causing a lesion of the carotid artery with consecutive development of an aneurysm spurium and rupture. Cerebral infarction, nevertheless, was the result of either occlusion or embolization.

Inadvertent arterial puncture during central venous cannulation of the internal jugular vein is not uncommon. Two reasons may be responsible for this: (i) the internal jugular vein lies in close proximity to the carotid artery (CA) and (ii) vessel anatomy is quite variable between individuals.

Although most of inadvertent arterial punctures fortunately remain without serious consequences, severe bleeding and/ or compression of the airways have been reported [3-6]. Routine response to carotid artery injury is to apply direct pressure. However, Reuber et al. [7] reported four cases with a stroke after IJVline insertion and carotid artery injury over a 6month period. They concluded that the true incidence of stroke is difficult to assess since its relation to IJV cannulation may be overlooked in patients who show no clear or immediate signs or in those who die. Cerebral ischemia is the most important symptom, which shows a wide range from transient ischemic attacks to massive stroke. Consciousness may be affected, but more often a focal neurological deficit is found (unilateral neurological signs such as aphasia, hemiparesis or hemiplegia). Therefore, any patient after an attempted, or successful internal jugular vein catheter insertion showing signs of cerebral ischemia should be investigated for a carotid artery lesion demanding contrast-enhanced computed tomography of the neck and head [7].

Beyond, arterial puncture morbidity increasing risks for severe bleeding and/ or development of an aneurysm spurium are coagulation disturbances and/ or an antiplatelet therapy [8-9]. Because accidental carotid artery cannulation can be potentially lethal, a vascular surgeon should be consulted before removal of a large bore dilator or catheter from the carotid artery, and exploration under controlled conditions should 
be considered [10]. This becomes especially important when dealing with atherosclerotic vessels.

Accordingly, the best approach of course is avoidance of inadvertent arterial puncture. We suggest the use of ultrasound for cannulation of the internal jugular vein, especially in patients receiving anticoagulants and those with atherosclerotic, kinked or stenotic carotid arteries. If arterial trauma with a large-caliber catheter occurs, prompt surgical or endovascular treatment seems to be the safest approach [10].

In conclusion, arterial puncture during central venous catheterization through the internal jugular vein still occur and may cause severe or even potentially life-threatening complications requiring surgical or interventional procedures to stop bleeding and re-establish compromised cerebral perfusion whenever jeopardized. Here, a low threshold for performing contrast enhanced a CT scan of neck and head is advisable.

\section{REFERENCES}

[1] Schummer W, Schummer C, Rose $\mathrm{N}$ et al. Mechanical complications and malpositions of central venous cannulations by experienced operators. A prospective study of 1794 catheterizations in critically ill patients. Intensive Care Med 2007; 33: 1055-1059.

[2] Schummer W, Köditz JA, Schelenz C et al. Preprocedure ultrasound increases the success and safety of central venous catheterization. $\mathrm{Br} \mathrm{J}$ Anaesth 2014; 113: 122-129.
[3] Heath KJ, Woulfe J, Lownie $\mathrm{S}$ et al. A devastating complication of inadvertent carotid artery puncture. Anesthesiology 1998; 89: 12731275.

[4] Schummer W, Schummer C. Another neurological complication of central venous cannulation. Eur J Anaesthesiol 2002; 19: 73-75.

[5] Benter T, Teichgraber UK, Kluhs L et al. Percutaneous central venous catheterization with a lethal complication. Intensive Care Med 1999; 25: 1180-1182.

[6] Ezri T, Szmuk P, Cohen Y et al. Carotid arteryinternal jugular vein fistula: A complication of internal jugular vein catheterization. J Cardiothorac Vasc Anesth 2001; 15: 231-232.

[7] Reuber M, Dunkley LA, Turton EP et al. Stroke after internal jugular venous cannulation. Acta Neurol Scand 2002; 105: 235-239.

[8] Oliver Jr WC, Nuttall GA, Beynen FM et al. The incidence of artery puncture with central venous cannulation using a modified technique for detection and prevention of arterial cannulation. J Cardiothorac Vasc Anesth 1997; 11: 851-855.

[9] Peces R, Navascues RA, Baltar J et al. Pseudoaneurysm of the thyrocervical trunk complicating percutaneous internal jugular-vein catheterization for hemodialysis. Nephrol Dial Transplant 1998; 13: 1009-1011.

[10] Guilbert MC, Elkouri S, Bracco D et al. Arterial trauma during central venous catheter insertion: Case series, review and proposed algorithm. J Vasc Surg 2008; 48: 918-925.

Citation: Wolfram Schummer, Samir G Sakka. Failed Internal Jugular Vein Cannulation - Is Contrast-Enhanced Computed Tomography Mandatory? ARC Journal of Anesthesiology. 2019; 4(2): 7-10. doi:dx.doi.org/ 10.20431/2455-9792.0402002.

Copyright: (C) 2019 Authors. This is an open-access article distributed under the terms of the Creative Commons Attribution License, which permits unrestricted use, distribution, and reproduction in any medium, provided the original author and source are credited. 